\title{
Direct Activation of Primary Motor Cortex during Subthalamic But Not Pallidal Deep Brain Stimulation
}

\author{
니ke A. Johnson, ${ }^{1 *}$ ๑ Jing Wang, ${ }^{1 *}$ @Shane D. Nebeck, ${ }^{1}$ Jianyu Zhang, ${ }^{1}{ }^{\circledR}$ Matthew D. Johnson, ${ }^{2}$ and Jerrold L. Vitek ${ }^{1}$ \\ Departments of ${ }^{1}$ Neurology and ${ }^{2}$ Biomedical Engineering, University of Minnesota, Minneapolis, Minnesota 55455
}

Deep brain stimulation (DBS) of the subthalamic nucleus (STN) and globus pallidus internus (GPi) is an effective treatment for parkinsonian motor signs. Though its therapeutic mechanisms remain unclear, it has been suggested that antidromic activation of the primary motor cortex (M1) plays a significant role in mediating its therapeutic effects. This study tested the hypothesis that antidromic activation of M1 is a prominent feature underlying the therapeutic effect of STN and GPi DBS. Single-unit activity in M1 was recorded using high-density microelectrode arrays in two parkinsonian nonhuman primates each implanted with DBS leads targeting the STN and GPi. Stimulation in each DBS target had similar therapeutic effects, however, antidromic activation of M1 was only observed during STN DBS. Although both animals undergoing STN DBS had similar beneficial effects, the proportion of antidromic-classified cells in each differed, 30 versus 6\%. Over $4 \mathrm{~h}$ of continuous STN DBS, antidromic activation became less robust, whereas therapeutic benefits were maintained. Although antidromic activation waned over time, synchronization of spontaneous spiking in M1 was significantly reduced throughout the $4 \mathrm{~h}$. Although we cannot discount the potential therapeutic role of antidromic M1 activation at least in the acute phase of STN DBS, the difference in observed antidromic activation between animals, and target sites, raise questions about its hypothesized role as the primary mechanism underlying the therapeutic effect of DBS. These results lend further support that reductions in synchronization at the level of M1 are an important factor in the therapeutic effects of DBS.

Key words: basal ganglia; deep brain stimulation; hyperdirect pathway; nonhuman primate; Parkinson's disease; primary motor cortex

Significance Statement

Recently there has been great interest and debate regarding the potential role of motor cortical activation in the therapeutic mechanisms of deep brain stimulation (DBS) for Parkinson's disease. In this study we used chronically implanted high density microelectrode arrays in primary motor cortex (M1) to record neuronal population responses in parkinsonian nonhuman primates during subthalamic nucleus (STN) DBS and globus pallidus internus (GPi) DBS. Our results suggest a contribution of antidromic activation of M1 during STN DBS in disrupting synchronization in cortical neuronal populations; however, diminishing antidromic activity over time, and differences in observed antidromic activation between animals and target sites with antidromic activation not observed during GPi DBS, raise questions about its role as the primary mechanism underlying the therapeutic effect of DBS.

\section{Introduction}

Deep brain stimulation (DBS) of the subthalamic nucleus (STN) and globus pallidus internus (GPi) is an effective surgical treat-

\footnotetext{
Received 0ct. 17, 2019; revised Jan. 23, 2020; accepted Jan. 28, 2020.

Author contributions: L.A.J., J.W., M.D.J., and J.L.V. designed research; L.A.J., J.W., S.D.N., and J.Z. performed research; L.A.J. and J.W. contributed unpublished reagents/analytic tools; L.A.J., J.W., S.D.N., and J.Z. analyzed data; L.A.J., J.W., and J.L.V. wrote the paper.

This work was supported by NIH NINDS: R01NS037019, R37NS077657, P50 NS098573, R01NS110613; and by MnDRIVE (Minnesota's Discovery, Research and Innovation Economy) Brain Conditions Program, Engdahl Family Foundation.

Conflicts of interest: J.L.V. serves as a consultant for Medtronic, Boston Scientific, and Abbott, and serves on the scientific advisory board for Surgical Information Sciences. The remaining authors declare no competing financial interests.

*L.A.J. and J.W. contributed equally to this work.

Correspondence should be addressed to Jerrold L.Vitek at vitek004@umn.edu.
}

ment for advanced Parkinson's disease (PD), though its therapeutic mechanisms remain unclear. Previous hypotheses that DBS inhibited output from the site of stimulation was based on the observation that surgical ablation and DBS in STN and GPi produced similar effects on motor signs in PD patients. Thus the concept that high-frequency electrical stimulation produced a functional lesion, inhibiting neuronal activity and reducing output from the stimulated nucleus (Benabid et al., 1987; Dostrovsky et al., 2000) became the prevalent early hypothesis of DBS mechanism of action. Subsequent studies demonstrated, however, that DBS increased the output from the stimulated nucleus via acti-

https://doi.org/10.1523/JNEUROSCI.2480-19.2020

Copyright $\odot 2020$ the authors 
vation of efferent axonal projections while also creating changes in firing patterns in downstream sites throughout the basalganglia thalamocortical network (Hashimoto et al., 2003; Xu et al., 2008; Vitek et al., 2012; Zhang et al., 2012; Agnesi et al., 2013a; Muralidharan et al., 2017). More recently attention has been focused on the impact of stimulation on axonal fibers projecting to and around the implanted nuclei. In line with this hypothesis several studies have proposed that STN DBS derives its efficacy via antidromic activation of the motor cortex (Gradinaru et al., 2009; Kuriakose et al., 2010; Li et al., 2012; Walker et al., 2012; Sanders and Jaeger, 2016; Anderson et al., 2018). Although the studies in support of this hypothesis have primarily investigated motor cortical responses that occur during STN DBS, via the "hyperdirect" corticosubthalamic pathway (Monakow et al., 1978; Nambu et al., 1996), it has also been suggested that similar effects may be elicited during GPi DBS (Li et al., 2014).

In humans putative antidromic activation has been evaluated through evoked potential recordings using scalp electroencephalogram or subdural electrocorticography (Walker et al., 2012; Miocinovic et al., 2018). This method provides an indirect measure of neuronal activity, however, and is susceptible to stimulation artifacts. A more reliable approach is to directly record from motor cortical neurons during stimulation. Experiments measuring single neuron responses have been conducted primarily in 6-OHDA lesioned rodents with stimulation targeting the STN (Gradinaru et al., 2009; Li et al., 2012; Sanders and Jaeger, 2016). Although these studies support antidromic activation of M1 during STN DBS in the rodent model of PD, antidromic activation during DBS in the nonhuman primate MPTP model, with head size and DBS leads that more appropriately scale to the human, has been unexplored. Moreover, it remains unclear whether similar neuronal responses can be observed during GPi DBS, which has been found to be similarly effective in treating motor signs in PD patients (Burchiel et al., 1999; Follett et al., 2010).

In this study we tested the hypothesis that antidromic activation of primary motor cortex (M1) neurons is a prominent feature of therapeutic STN and GPi DBS by evaluating single-unit activity from high-density microelectrode arrays chronically implanted in the M1 area of two parkinsonian nonhuman primates. In this study we also explored an alternative hypothesis that proposes DBS exerts its therapeutic effects by modifying the pattern of synchronized activity in M1. These studies were performed in the same animals providing direct within-subjects comparison of these two hypotheses. Each animal was implanted with two scaled-down versions of human DBS leads targeting the STN and $\mathrm{GPi}$, respectively, enabling within animal comparisons of M1 activity to stimulation of both nuclei. Whereas previous unit physiology studies were limited to examining acute effects of DBS on the timescale of minutes, we examined spontaneous firing during STN DBS over the course of $4 \mathrm{~h}$ while assessing motor signs before, at hourly intervals during, and following DBS to examine the stability of the observed effects.

\section{Materials and Methods}

\section{Surgical procedures}

All procedures were approved by the University of Minnesota Institutional Animal Care and Use Committee and complied with U.S. Public Health Service policy on the humane care and use of laboratory animals. Two adult female rhesus macaques [Macaca mulatta, subjects: J (17 years), K (13 years)] were used in this study. Surgeries were performed under isoflurane anesthesia using aseptic techniques. Surgical planning of a titanium cephalic chamber targeting the STN and GPi was done using merged preoperative cranial CT and MRI images in the Monkey Cicerone neurosurgical navigation program (Miocinovic et al., 2007).
A

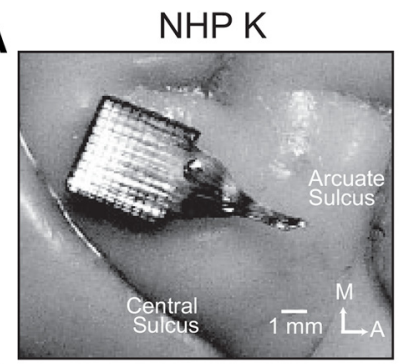

B


Figure 1. Location of Utah array and DBS leads. $A$, Utah arrays were placed over the arm area of the M1 (top). The image of Subject $K$ was obtained end of study after perfusion; the image of Subject I was obtained intraoperatively. B, DBS lead locations in the STN and GPi with location of DBS leads in the STN and GPi in each subject. Contacts used for therapeutic stimulation shown in yellow. In both animals, we verified that DBS in STN and GPi contacts produced improvements in parkinsonian motor signs (Table 1). Th = thalamus, $\mathrm{SN}=$ substantia nigra, $\mathrm{GPe}=$ globus pallidus externus.

Methods for DBS implantation are described in detail in a previous publication (Hashimoto et al., 2003). Briefly, the location of the target nuclei was confirmed using extracellular microelectrode mapping and each animal was implanted targeting the STN and GPi with an 8-contact scaleddown version of human DBS leads $(0.5 \mathrm{~mm}$ contact height, $0.5 \mathrm{~mm}$ inter-contact spacing, $0.625 \mathrm{~mm}$ diameter; NuMed). In a subsequent surgery, each animal was implanted in the arm area of the M1 with a 96-channel Utah microelectrode array (Pt-Ir, $1.5 \mathrm{~mm}$ depth, $400 \mu \mathrm{m}$ inter-electrode spacing; Blackrock Microsystems) using surgical methods described previously (Rousche and Normann, 1992; Maynard et al., 1997). M1 was identified based on sulcal landmarks during the array implantation surgery, and the arm area was localized based on intraoperative stimulation of the cortical surface using a stainless-steel ball electrode (Grass Technologies). Locations of DBS leads were verified in Subject K histologically using frozen coronal sections (50 $\mu \mathrm{m}$ thick) that were imaged and visualized in the sagittal plane in Avizo 3D analysis software (FEI; Fig. 1B, left). Histology is not yet available in Subject J; however, DBS lead locations were approximated using fused preimplantation MRI and post-implantation CT images (Fig. $1 B$, right) together with microelectrode identification of the target nuclei before implantation of the leads.

\section{MPTP administration and behavioral assessments}

Animals were rendered parkinsonian by systemic (intramuscular) and intra-carotid injections of the neurotoxin 1-methyl-4-phenyl-1,2,3,6 tetrahydropyridine (MPTP). Subject K received six intramuscular injections $(0.3-0.4 \mathrm{mg} / \mathrm{kg}$ each, total $1.8 \mathrm{mg} / \mathrm{kg})$. Subject J received three intramuscular injections and one intra-carotid injection $(0.3-0.4 \mathrm{mg} / \mathrm{kg}$ each, total $1.4 \mathrm{mg} / \mathrm{kg}$ ). For both subjects, data were gathered after a stable parkinsonian state was achieved, $\sim 2$ months after the last MPTP injection. Overall parkinsonian severity was assessed using a modified Unified Parkinson's Disease Rating Scale (mUPDRS), which rated axial motor symptoms (gait, posture, balance, turning, defense reaction, and food retrieval) as well as appendicular motor symptoms (upper and lower limb rigidity, bradykinesia, akinesia, and tremor) on the hemi-body contralateral to neural recordings using a $0-3$ scale $(0=$ normal, $3=$ severe $)$. 
Table 1. Improvement in parkinsonian motor signs during acute STN and GPi DBS

\begin{tabular}{lllcc}
\hline Subject & Motor sign & $\begin{array}{l}\text { mUPDRS } \\
\text { baseline }\end{array}$ & $\begin{array}{l}\text { STN-DBS } \\
\text { improvement, } \%\end{array}$ & $\begin{array}{l}\text { GPi-DBS } \\
\text { improvement, \% }\end{array}$ \\
\hline $\mathrm{K}$ & Rigidity & $2.0(2.0,2.2)$ & $53(53,58)$ & $50(44,54)$ \\
& Tremor & $0.5(0.5,0.5)$ & $100(100,100)$ & $100(100,100)$ \\
& Bradykinesia & $2.5(2.0,3.0)$ & $50(50,50)$ & $42(33,50)$ \\
& Akinesia & $2.5(2.0,3.0)$ & $50(50,50)$ & $50(50,50)$ \\
$\mathrm{J}$ & Food retrieval & $2.5(2.5,2.5)$ & $50(35,62)$ & $40(40,40)$ \\
& Rigidity & $0.9(0.9,1.1)$ & $47(37,55)$ & $47(40,54)$ \\
& Tremor & $0.0(0.0,0.0)$ & $\mathrm{NA}$ & $\mathrm{NA}$ \\
& Bradykinesia & $2(1.5,2)$ & $33(25,50)$ & $33(25,50)$ \\
& Akinesia & $2(1.5,2)$ & $33(25,50)$ & $33(25,50)$ \\
& Food retrieval & $2(1.5,2)$ & $25(25,33)$ & $33(25,35)$ \\
\hline
\end{tabular}

Values are medians (1st quartile, 3rd quartile). mUPDRS for NHPs was used to assess motor signs in each animal, rating upper limb rigidity, tremor, bradykinesia, akinesia, and food retrieval (on the arm contralateral to neural recordings) using a $0-3$ scale ( $0=$ normal, $3=$ severe). Baseline Off-DBS scores are shown, as are percentage improvement observed with STN and GPi DBS. Number of observations: Subject K, baseline $n=23$, Off- versus STN DBS and Off- versus GPi DBS within-session comparisons $n=10$ and 6 , respectively. Subject J, baseline $n=38,0 \mathrm{ff}-$ versus STN DBS and Off versus GPi DBS within-session comparisons $n=17$ for each. There was no significant difference between median percentage improvement in motor signs with STN versus GPi DBS in either animal (Wilcoxon rank sum, $p>0.05$, since all $p$ values were above 0.05 .

During physiology recording sessions animals were seated in a primate chair, therefore therapeutic effects of DBS were assessed based on appendicular motor scores only. Subject K received daily dopaminergic treatment (carbidopa/levodopa 25/100 mg tablets) at the end of the day's experimental sessions to facilitate the animal's care in its home enclosure. To minimize possible carryover effects of levodopa on physiological recordings, all recording sessions were conducted a minimum of $16 \mathrm{~h}$ after the last dose.

\section{Neuronal recordings}

Neurophysiological data were collected using a TDT workstation (Tucker Davis Technologies) operating at $\sim 25 \mathrm{kHz}$ sampling rate. Spontaneous activity of $\mathrm{M} 1$ neurons was recorded while the animal was seated, head fixed, in a primate chair. Unless noted otherwise only time periods in which the animal's eyes were open and the arm contralateral to the recording site was not moving were included in the analysis, as described in the following paragraphs.

Video of the animal's eyes was captured and synchronized to the physiological recordings. Image segmentation algorithms developed in MATLAB (MathWorks) and described in a previous publication (Escobar Sanabria et al., 2017) were used to analyze video of one eye and calculate the eye-open area in each video frame. Areas were normalized by the eye-open area identified when the animal had the eyes wide open, such that a value of one corresponds to eye fully open, zero closed. Time periods with eye-open area below a threshold of 0.5 were excluded from further analysis.

Arm movements were recorded using a passive marker video capture system (Motion Analysis), which tracked and digitally recorded in 3D the location of a reflective marker on the animal's contralateral wrist $(100 \mathrm{~Hz}$ sampling rate). The wrist position data were smoothed with a 100sample moving average filter and the derivative taken to obtain wrist speed. Time periods with wrist speeds above a threshold of $5 \mathrm{~mm} / \mathrm{s}$, plus $0.5 \mathrm{~s}$ before and after threshold crossings, were excluded from analysis. Only time periods $>5 \mathrm{~s}$ in which eyes were open and no arm movement was detected were included in subsequent analysis.

\section{DBS stimulation protocol}

Two sets of stimulation conditions were acquired in each animal: acute DBS, which lasted 1-2 min, and subacute DBS, which lasted $4 \mathrm{~h}$. The two conditions were collected on separate days.

Acute DBS was delivered using TDT current-controlled stimulator $(\mathrm{IZ2H})$ connected to the DBS lead contacts. Pulse rate and pulse width were constrained by the sampling rate of the TDT system and were set to 132.7 Hz and $81.9 \mu$ s, respectively (balanced biphasic pulses, no interphase gap). Therapeutic stimulation amplitude and bipolar contact pairs that produced improvement in parkinsonian motor signs were identified (Fig. 1, bottom; Table 1) and used in subsequent acute DBS recording sessions. The stimulation protocols used in the acute STN DBS sessions were Subject $\mathrm{K}, \mathrm{C} 1(+), \mathrm{C} 2(-)$, at $0.3 \mathrm{~mA}$ amplitude; Subject J, C0(-), $\mathrm{C} 2(+)$, at $0.425 \mathrm{~mA}$ amplitude. Acute GPi DBS parameters were Subject $\mathrm{K}, \mathrm{C} 0(-), \mathrm{C} 1(+)$, at $0.8 \mathrm{~mA}$ amplitude; Subject J, C3(-), C4(+), at 0.6 $\mathrm{mA}$ amplitude. STN and GPi DBS for each animal were delivered on separate days.

Subacute DBS was delivered using clinically implantable pulse generators [IPGs; Subject K: St. Jude (now Abbott) Medical; Subject J: Medtronic] externally connected to the STN DBS lead contacts. Subacute GPi DBS was not examined in this study. Therapeutic stimulation amplitude using the same contacts used for the acute DBS with the IPGs was identified. The stimulation protocols used in the $4 \mathrm{~h}$ stimulation and recording sessions were Subject $\mathrm{K}: \mathrm{C} 1(+), \mathrm{C} 2(-), 125 \mu$ s pulse width, $130 \mathrm{~Hz}$ pulse rate, and $0.2 \mathrm{~mA}$ amplitude; Subject J: C0(-), C2(+),120 $\mu$ s pulse width, $130 \mathrm{~Hz}$ pulse rate, and $2.1 \mathrm{~V}$ amplitude. In each animal subacute DBS was delivered $4 \mathrm{~h}$ per day for 5 consecutive days.

\section{Neuronal data analysis}

Neuronal recordings were analyzed offline using custom software developed in MATLAB (MathWorks) and Offline Sorter (Plexon). Raw data were bandpass filtered $300-5000 \mathrm{~Hz}$, and single units were isolated and sorted using principal component and template-based methods in Offline Sorter. To remove the possibility of spurious detection of electrical artifacts as spikes, data between $0.2 \mathrm{~ms}$ before and $0.8 \mathrm{~ms}$ after each DBS pulse timestamp was excluded from the analysis. Similar data blanking was performed to the off-DBS datasets using virtual pulse timestamps introduced at the same frequency as that used during DBS.

Peristimulus time histograms (PSTH; bin size $=0.2 \mathrm{~ms}$ ) of neuronal activity were examined triggered to either the stimulation pulses during DBS or to virtual stimulation pulses off-DBS. All spike occurrences in each bin were summed, and divided by the total number of pulses to get a probability PSTH, and divided again by the bin size to get a firing rate PSTH. For within-cell comparisons of the effect of DBS on firing rates, firing rate PSTHs were calculated in $2 \mathrm{~s}$ epochs in each condition to obtain a distribution of firing rates for each cell (e.g., 60 firing rate values over a 2 min recording period). The on-DBS firing rate distribution was compared with the off-DBS distribution to assess whether DBS significantly increased or decreased the firing rate of a given cell (Wilcoxon rank sum test, $p<0.05$ ).

Cells were classified as antidromic based on whether short latency, low temporal jitter, high firing activity was detected following the DBS pulses (Fig. 2B). It should be noted that classification is based solely on the PSTH, and collision experiments were not conducted to confirm that DBS-evoked activity was definitively antidromic. The firing rate in each PSTH bin was converted to a $z$-score relative to baseline firing rate bins taken from the off-DBS period, based on a PSTH triggered to virtual stimulation pulses. If there was a peak in the resulting PSTH with a maximum $z$-score $>10$, latency $<3 \mathrm{~ms}$, and low temporal jitter (width at half-maximum $\leq 3$ bins), the stimulation evoked activity was classified as antidromic.

\section{Spike synchronization analysis}

Spike synchronization across simultaneously recorded cells in the M1 array was calculated using the source codes developed by Thomas Kreuz's group (http://wwwold.fi.isc.cnr.it/users/thomas.kreuz/) and the method (Kreuz et al., 2015; Mulansky et al., 2015) is briefly described here. This measure quantifies the degree of synchrony of the simultaneously recorded unit activities by detecting coincidences of spikes appearing quasi-simultaneously. First, we defined a spike train as $\left\{t_{i}\right\}$, $t_{i}<t_{i+1}$, with $t_{i}$ representing the times of the spikes. For $N$ simultaneously recorded spike trains $\left\{t_{i}^{(1)}, t_{i}^{(2)}, \ldots t_{i}^{(N)}\right\}$, a generalized bivariate coincidence index for all pairs of spike trains was calculated using a coincidence indicator:

$$
C_{i}^{(n, m)}=\left\{\begin{array}{c}
1 \text { if } \min _{j}\left(\left|t_{i}^{(n)}-t_{j}^{(m)}\right|<\tau_{i j}^{(n, m)}\right) \\
0 \text { otherwise }
\end{array},\right.
$$

with coincidence window $\tau$ adapted to the local firing rates:

$$
\tau_{i j}^{(n, m)}=\min \left\{t_{i+1}^{(n)}-t_{i}^{(n)}, t_{i}^{(n)}-t_{i-1}^{(n)}, t_{j+1}^{(m)}-t_{j}^{(m)}, t_{j}^{(m)} t_{j-1}^{(m)}\right\} / 2,
$$


A

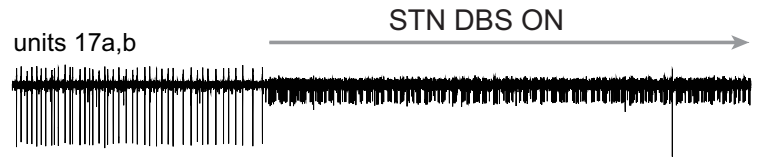

unit $75 a$

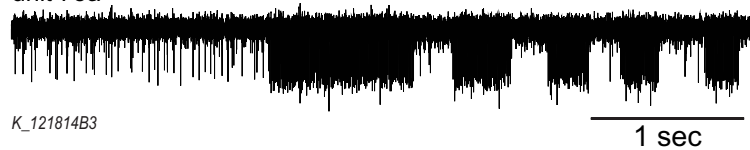

K_121814B3

B


Excitation

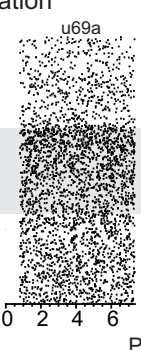

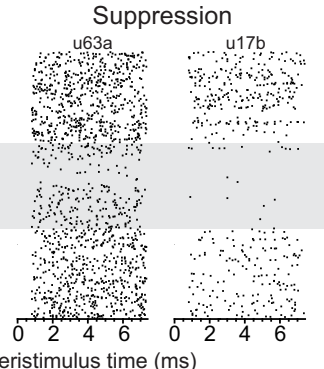

Suppression
1 sec
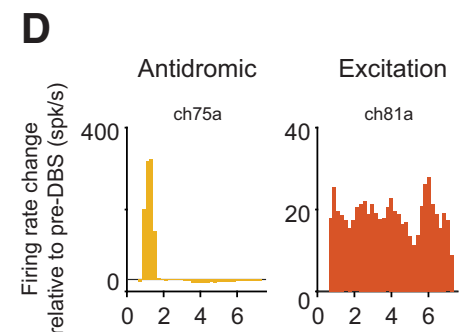

Peristimulus time (ms)



Acute GPi DBS

Acute STN DBS
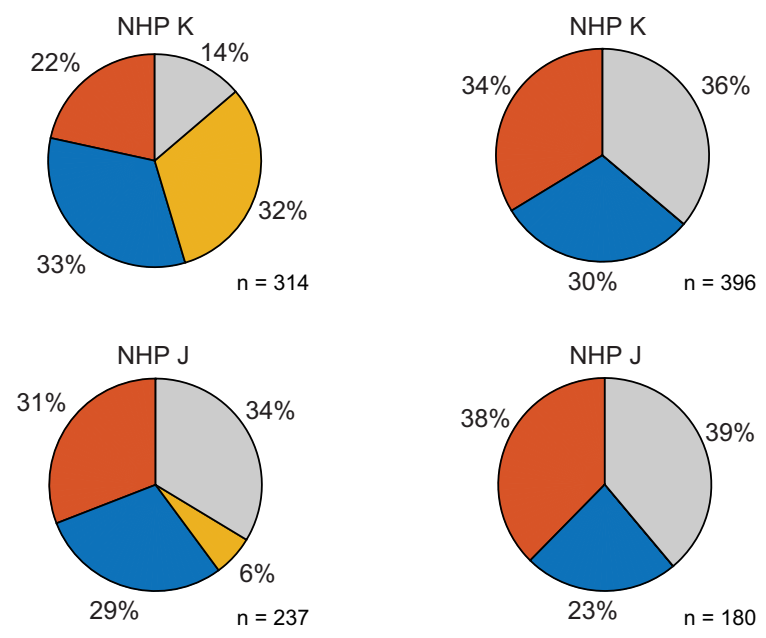

Antidromic
No Change

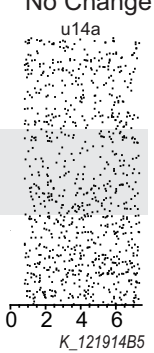

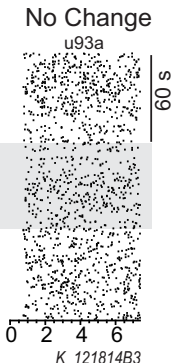
(n)

Figure 2. M1 single-unit responses to acute STN and GPi DBS. $A$, Example recording traces from two channels at the onset of STN DBS. Channel 17 had two isolated cells: 17 a decreased firing rate and $17 \mathrm{~b}$ increased firing rate. Channel 75 had one isolated cell with antidromic firing response. $\boldsymbol{B}$, Examples of peristimulus time raster plots aligned to DBS (or sham) pulses before, during, and after STN DBS illustrating the four main response categories based on firing rate and pattern: antidromic, suppression, excitation, and no change. C, Examples of peristimulus time raster plots demonstrating suppression, excitation, and no change responses before, during, and after GPi DBS. Antidromic activation of M1 was not observed with GPi DBS. D, Normalized peristimulus time histograms (firing rate relative to the mean pre-DBS period) are shown, illustrating the four response classifications used in this study. $\boldsymbol{E}$, Pie charts illustrating the percentage of each response type in M1 during acute STN (left) and GPi DBS (right) for each animal. Antidromic firing in M1 was only observed during STN DBS, and in greater proportion in Subject K than in Subject J, whereas similar proportions of excitation and suppression were observed for each animal for either STN or GPi DBS.

and averaged for each spike in every spike train:

$$
C_{i}^{(n)}=\frac{1}{N-1} \sum_{m \neq n} C_{i}^{(n, m)}
$$

The spike synchronization score was then defined as the ratio for coincident spikes:

$$
S Y N C=\frac{\sum C}{M},
$$

where $M$ was the total number of spikes in $N$ spike trains. Spike synchronization score is zero if and only if the spike trains do not contain any coincidences and reaches one if and only if each spike in every spike train has one matching spike in all other spike trains (Kreuz et al., 2015; Mulansky et al., 2015).

The time frames with animals awake, at rest, without movement were identified using the motion and reference video data. M1 spike train data during these time frames was divided into $500 \mathrm{~ms}$ bins. A spike synchronization score was calculated for each bin and all the scores from each recording were pooled. The distributions of the scores from different conditions (e.g., pre-, during, and post-DBS) were then compared to investigate the change of the synchronization level of M1 neuronal activity with STN DBS.

Pairwise spike synchronization was also calculated for each pair of cells and presented as spike synchronization matrixes. In the matrix, the color of each pixel represents the value of the synchronization score (warmer = higher synchronization score) of one pair of spike trains using the above functions in the condition where $N=2$. Whereas the population spike synchronization score takes into account all simultaneously recorded cells, this pairwise analyses was used to explore whether synchronization between different groups of cells was altered during DBS. The cell-cell pairs were separated into three groups: antidromic to antidromic (Anti-Anti), antidromic to non-antidromic (Anti-NonAnti), and non-antidromic to non-antidromic (NonAnti-NonAnti) neurons.

\section{Statistical analysis}

$m U P D R S . t$ Test $[t(\mathrm{DoF})]$ was used to compare mUPDRS scores in the DBS On versus Off condition for both the STN and GPi for Subjects K and $\mathrm{J}$, individually. Wilcoxon test $\left[\chi^{2}(\mathrm{DoF}, N)\right]$ was used to evaluate the difference between the mUPDRS of individual symptoms ON/post $4 \mathrm{~h}$ STN DBS and that pre-DBS. Bonferroni correction for five parkinsonian signs (rigidity, tremor, bradykinesia, akinesia, and food retrieval) was applied for both tests.

Spike synchronization analysis. One-way ANOVA test $[F(\mathrm{DoF}, N)]$ was used to detect changes in spike synchronization before, during (first, second, third, and fourth hour on), and after (first and second hour post) $4 \mathrm{~h}$ STN DBS for individual sessions. Multivariate mixed-model ANOVA test $[F(\operatorname{DoF}, N)]$ was used for the spike synchronization from multiple sessions combined, defining the ON/OFF condition as the fixed effect 
and different sessions \{condition $\}$ as the random effect. Thereafter, Dunnett's test was used for both the spike synchronization from an individual session and all sessions combined to evaluate the difference between each condition during/post-4 h STN DBS and the pre-DBS condition.

\section{Results}

Therapeutic effects and M1 cell activity during acute DBS STN and GPi DBS were effective in reducing parkinsonian motor signs including rigidity, bradykinesia, and akinesia in both animals (Table 1). Tremor is typically minimal or not observed in the rhesus macaque MPTP model, though Subject K, which exhibited more severe parkinsonian motor signs, had mild tremor that was alleviated by acute STN and GPi DBS. The median percentage improvement in mUPDRS scores with STN DBS compared with GPi DBS was not significantly different in either animal for any of the motor signs evaluated (Wilcoxon rank sum test, $p>0.05$ ).

The effect of DBS on M1 spiking activity was not homogenous across the population of recording cells (Subject J: $n=314$ STN, $n=237$ GPi; Subject K: $n=396$ STN, $n=180$ GPi.). This is well illustrated by Figure $2 A$, top recording trace, which contains two isolated cells; one cell had a dramatic suppression of firing after the onset of STN DBS, whereas the other cell increased firing during stimulation. Figure $2, B$ and $C$, raster plots, show five representative cell responses to STN and GPi DBS, respectively. The effect of DBS on cell firing was classified as antidromic, excitation, suppression, or no change based on the firing rate PSTHs before and during DBS (Fig. 2D; see Materials and methods). GPi DBS evoked excitatory, suppressive, and no change responses in similar proportions between animals (Fig. 2E, right). Antidromic responses to DBS, characterized by action potentials occurring at short latency and low temporal jitter after stimulation pulses, were only observed during STN DBS. Antidromic activity in M1 during STN DBS was observed in both animals, however there was a large difference between animals in the proportion of antidromic classified cells (Fig. 2E, left). In Subject K nearly one-third of all cells were classified as antidromic, whereas in Subject J only $6 \%$ of recorded cells were found to have antidromic activity. The mean \pm SD latency of antidromic firing across all antidromic classified cells was $1.23 \pm 0.43 \mathrm{~ms}$.

In summary, although improvement in clinical ratings were similar with STN and GPi DBS, antidromic activity in M1 was only observed during STN DBS. It is also important to note that although the therapeutic effect of STN DBS in each Subjects K and J was similar (Table 1), the proportion of cells demonstrating antidromic activation were significantly different (32 vs $6 \%, \chi^{2}$ test, $\chi^{2}=54.39, p<0.001$ ). The following sections focus on the characteristics of antidromic classified cells in the context of acute and subacute $(4 \mathrm{~h})$ STN DBS.

\section{Time course of antidromic activity: acute STN DBS}

As illustrated by the recording trace in Figure $2 \mathrm{~A}$ (bottom plot), antidromic classified cells did not discharge in response to every stimulation pulse. We observed many cells showing epochs of antidromic activity followed by pauses in firing during stimulation. We also observed that the robustness of antidromic discharges decreased over time. The time course of the population averaged PSTH of antidromic classified cells after the onset of STN DBS is shown in Figure 3A. The majority of cells demonstrated a significant decrease in firing rate over the first $50 \mathrm{~s}$ of STN DBS (Fig. 3B), suggesting that antidromic firing in M1 during STN DBS is not stationary and tends to decrease over time.

We also characterized antidromic activity with lower frequency pulse rates in a subset of neurons in Subject $\mathrm{K}(n=23)$.
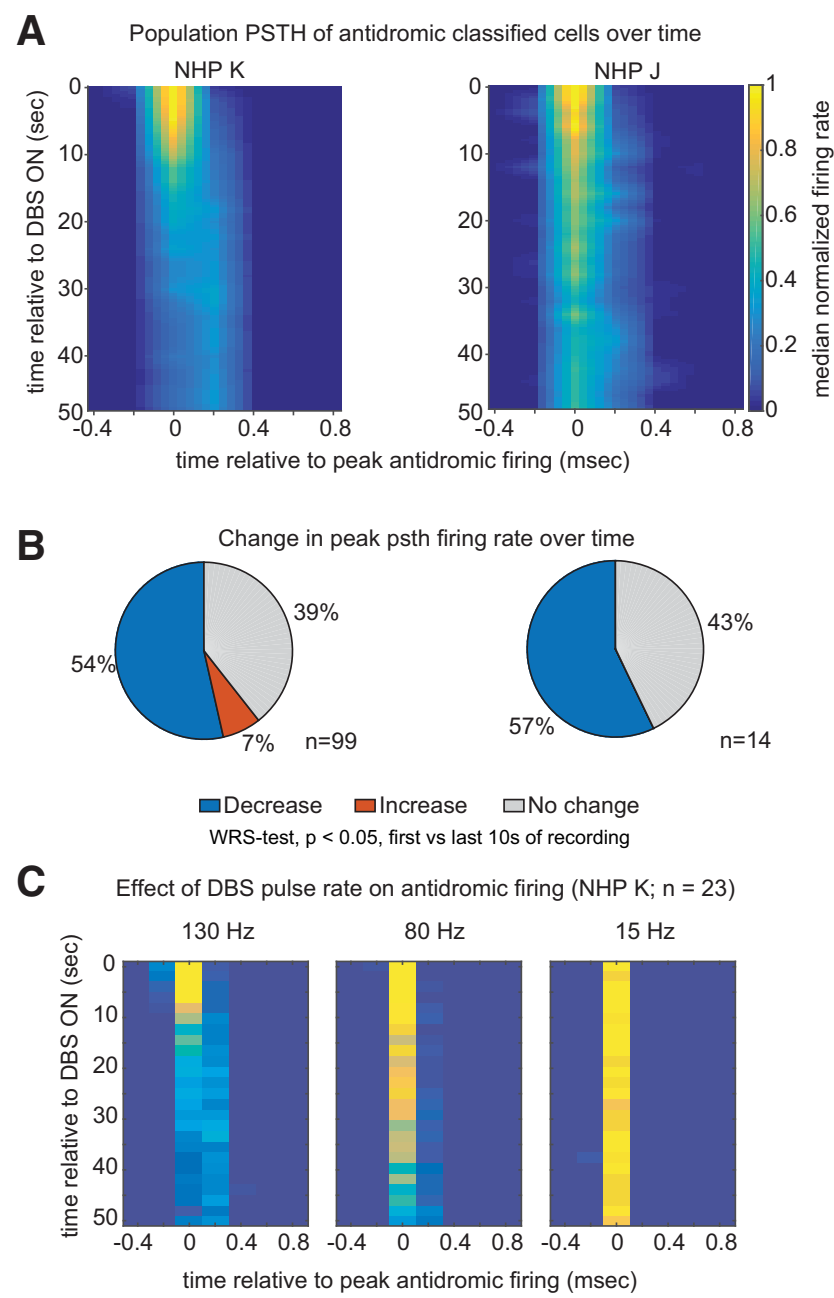

Figure 3. Strength of antidromic firing in M1 diminishes over time. $\boldsymbol{A}$, Heat map illustrating the time course of the population PSTH of M1 cells that were antidromically activated during the first 50 s of STN DBS for both subjects. Before averaging, the PSTH of each cell was aligned to the time bin in the PSTH where firing was maximal, and firing rate normalized to the firing rate calculated over the first three time bins. Zero on the $x$-axis represents the time point of peak antidromic firing. PSTH were calculated in $2 \mathrm{~s}$ bins and for visualization a 5 -point linear interpolation both $x$ and $y$ axes was performed. $\boldsymbol{B}$, The percentage of cells with significant change in their peak PSTH firing rate [first $10 \mathrm{~s}$ vs the last $10 \mathrm{~s}$, Wilcoxon rank sum (WRS) test, $p<0.05$ ]. C, Time course of the population PSTH of cells whose responses to 15 and $80 \mathrm{~Hz}$ stimulation were tested in addition to $130 \mathrm{~Hz}$.

The trend of decreased antidromic firing over time appears to be characteristic of more rapid pulse rates; whereas the robustness of antidromic firing during 130 and $80 \mathrm{~Hz}$ stimulation decreased over the first $50 \mathrm{~s}$ of recording, during $15 \mathrm{~Hz}$ stimulation antidromic firing persisted (Fig. 3C).

\section{Therapeutic effects and time course of antidromic activity: subacute $4 \mathrm{~h}$ STN DBS}

In another set of experiments animals were provided with therapeutic STN DBS continuously for $4 \mathrm{~h}$ for 5 consecutive days (see Materials and Methods). Within each day the improvement in composite mUPDRS scores, collected in Hours 1-3, was stable throughout the period of stimulation for both animals (Fig. $4 A, C$ ), though some variability across days was observed particularly in Subject J. Effects of STN DBS on individual motor signs is shown in Table 2. In Subject K, the percentage improvement ranged from 30 to $45 \%$ and was significant for rigidity, bradyki- 

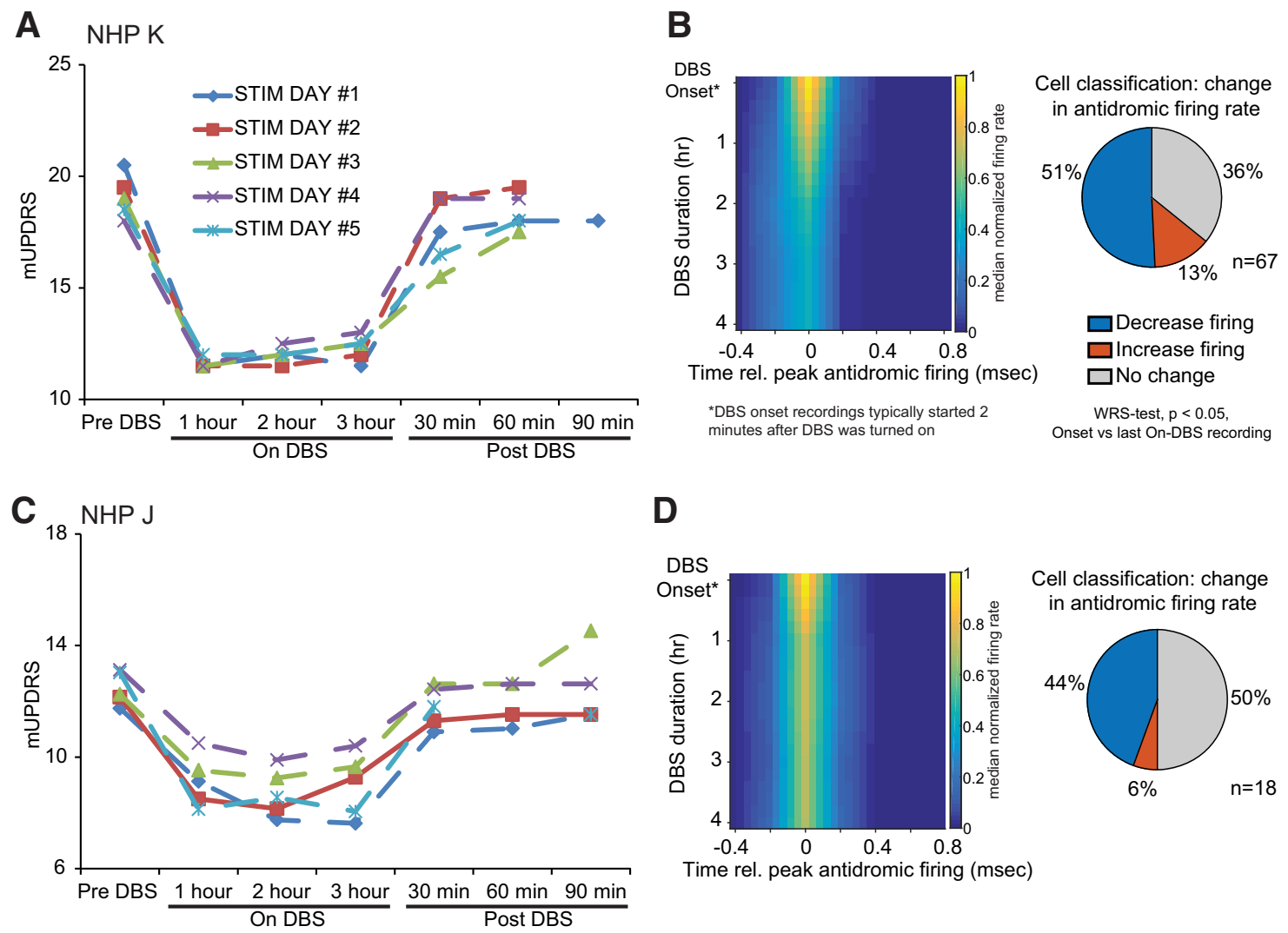

D

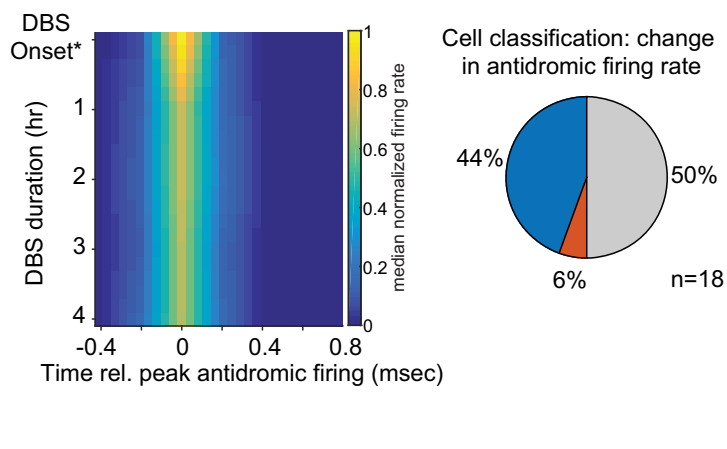

Figure 4. The strength of antidromic activation of M1 cells was progressively diminished over $4 \mathrm{~h}$ of continuous STN. $A, C$, Composite scores based on the mUPDRS ( $y$-axis) for Subjects $K$ and J, respectively. $X$-axis represents the time of stimulation. mUPDRS scores are shown for Hours 1-3 but were not taken in Hour 4. Detailed scores for individual motor signs are presented in Table 2. $\boldsymbol{B}$, $D$, Time course of cell population PSTH over $4 \mathrm{~h}$ of STN DBS in each animal is shown in the heat map (left). The percentage of cells with significant change in their peak PSTH firing rate [DBS onset vs $4 \mathrm{~h} \mathrm{DBS}$ duration, Wilcoxon rank sum (WRS) test, $p<0.05$ ] is shown in the pie charts (right). PSTH were calculated in 2 s bins and grouped into DBS onset (first $50 \mathrm{~s}$ of recording) and $1,2,3$, and $4 \mathrm{~h}$ DBS time bins. Before averaging, the PSTH of each cell was aligned to the time bin in the PSTH where firing was maximal, and firing rate normalized to the firing rate calculated over the first three time bins.

Table 2. Clinical ratings of parkinsonian animals before, during and after subacute STN DBS

\begin{tabular}{|c|c|c|c|c|c|c|c|c|}
\hline \multirow[b]{2}{*}{ Subject } & \multirow[b]{2}{*}{$\begin{array}{l}\text { Motor sign } \\
\text { (highest possible score) }\end{array}$} & \multirow[b]{2}{*}{ Pre-DBS } & \multicolumn{3}{|l|}{ On-DBS } & \multicolumn{2}{|l|}{ Post-DBS } & \multirow[b]{2}{*}{$\begin{array}{l}\text { Highest improvement } \\
\text { with DBS, \% }\end{array}$} \\
\hline & & & $1 \mathrm{~h}$ & $2 \mathrm{~h}$ & $3 \mathrm{~h}$ & $30 \mathrm{~min}$ & $60 \mathrm{~min}$ & \\
\hline \multirow[t]{4}{*}{ K } & *Rigidity (6) & $6.0(4.8,6.0)$ & $3.5(3.5,4.0)$ & $3.5(3.5,4.3)$ & $3.5(3.5,3.8)$ & $5.0(4.8,5.8)$ & $5.5(5.0,6.0)$ & 42 \\
\hline & *Bradykinesia (6) & $5.0(5.0,5.5)$ & $3.5(3.0,3.5)$ & $3.5(3.0,3.5)$ & $3.5(3.5,3.5)$ & $5.0(4.3,5.0)$ & $4.5(4.5,5.0)$ & 30 \\
\hline & *Akinesia (6) & $5.5(5.5,5.5)$ & $3.0(2.5,3.3)$ & $3.0(2.8,3.8)$ & $3.0(2.8,3.8)$ & $4.5(4.0,5.3)$ & $5.0(5.0,5.5)$ & 45 \\
\hline & ${ }^{*}$ Food retrieval (3) & $2.5(2.5,2.5)$ & $1.5(1.0,1.5)$ & $1.5(1.0,1.5)$ & $1.5(1.0,2.0)$ & $2.5(2.5,2.5)$ & $2.5(2.5,2.5)$ & 40 \\
\hline \multirow{3}{*}{ J } & *Bradykinesia (6) & $4.0(3.8,4.0)$ & $3.0(2.8,3.5)$ & $2.5(2.5,3.5)$ & $3.5(2.5,3.5)$ & $4.0(3.8,4.0)$ & $4.0(3.6,4.0)$ & 38 \\
\hline & ${ }^{*}$ Akinesia (6) & $4.0(4.0,4.0)$ & $2.5(2.5,3.3)$ & $3.0(2.3,3.0)$ & $3.0(2.5,3.3)$ & $4.0(3.5,4.0)$ & $4.0(3.6,4.0)$ & 38 \\
\hline & Food retrieval (3) & $2.0(2.0,3.0)$ & $2.0(1.5,2.3)$ & $2.0(1.5,2.3)$ & $1.5(1.5,2.3)$ & $2.5(1.8,2.5)$ & $2.3(1.6,2.5)$ & 25 \\
\hline
\end{tabular}

Values are medians (1st quantile, 3rd quantile). The number of assessments was five for each condition except for the values in italic, which were four. On DBS ratings were not taken in Hour 4.

${ }^{*} p<0.05$, Wilcoxon test. Bold scores are significantly different from its Pre-DBS condition in Steel's test with control $=$ Pre-DBS.

nesia, akinesia, and food retrieval. In Subject J, percentage improvement ranged from 25 to $45 \%$ and was significant for rigidity, bradykinesia, and akinesia.

Although the therapeutic effects of STN DBS remained relatively stable over the $4 \mathrm{~h}$ period of stimulation, the robustness of antidromic firing decreased over time in 51\% $(n=34 / 67)$ and $44 \%(n=8 / 18)$ of cells in Subjects K and J, respectively (Fig. $4 B, C)$ continuing the trend observed with acute DBS (Fig. 2). It should be noted that the subacute STN DBS neural recordings began $\sim 2$ min after the DBS pulse generator was turned on, such that the reductions in antidromic firing described during acute
DBS would already be occurring when these subacute recordings began. The change in antidromic firing rate was most salient in Subject K, which had a higher proportion of antidromic classified cells compared with Subject J. These findings further demonstrate that although motor benefit is maintained antidromic firing in M1 due to STN DBS tends to decrease over time.

Combining all recording sessions, we found no statistically significant correlation between the clinical ratings and normalized antidromic firing rates across the cell population in Subject $\mathrm{K}$ (Spearman's rank correlation, $r_{s}=0.04, p=0.63$ ), and a weak correlation in Subject J $\left(r_{s}=-0.3, p=0.03\right)$. Within individual 
A

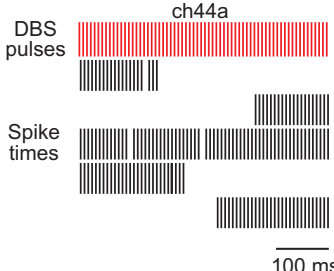

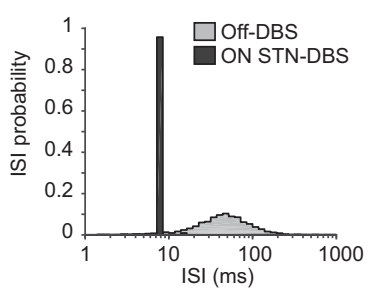

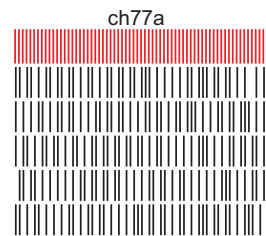

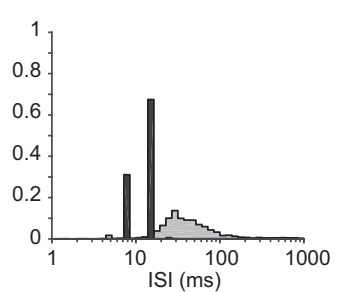

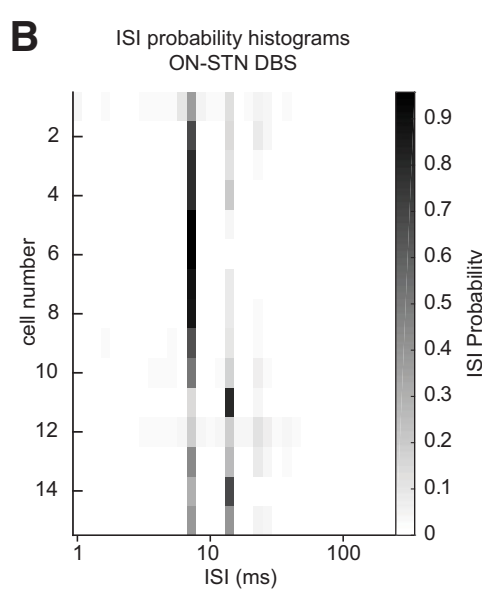

C


Figure 5. Interspike interval (ISI) probability histograms illustrate that antidromic-classified cells had highly regular discharge patterns during DBS. A, Spike occurrences during STN DBS in two $\mathrm{M} 1$ cells from Subject Kare shown in the top ( $2.5 \mathrm{~s}$ duration segment shown, each row $=0.5 \mathrm{~s}$ ). Corresponding ISI probability histograms from the entire recording block are shown in the bottom, with Off-DBS ISI histograms shown for comparison. Prominent peaks corresponding to one and two times the DBS stimulation interstimulus period ( $\sim 7.7 \mathrm{~ms})$ are evident. $\boldsymbol{B}$, ISI probability histograms from 15 simultaneously recorded cells identified as antidromic (Subject K) displayed as a heat map. C, Proportion of antidromic-classified cells in subacute DBS sessions with ISI probability peaks at one or two times the DBS ISI (or other) based on recordings at early $(<0.5 \mathrm{~h}$ on-DBS) and late (3- $4 \mathrm{~h}$ on-DBS) in the on-DBS recording period.

sessions, there was no statistically significant correlation between the clinical ratings and antidromic firing across the cell population in either animal (Spearman's rank correlation, $p>0.05$ ).

\section{Discharge patterns of antidromic classified cells is not Poisson-like}

Prior studies have suggested that STN DBS produces antidromic firing in M1 that is stochastic and Poisson-like (Li et al., 2012). To characterize the discharge patterns of antidromic classified cells, interspike interval histograms were created (Fig. 5A). Although a large percentage of individual DBS pulses failed to elicit antidromic spikes, the resulting pattern cannot be considered Poisson-like; we found that most antidromic-classified cells had interspike interval histograms with peaks equal to the DBS interstimulus interval (Fig. $5 B, C$ ). Although overall the robustness of antidromic firing reduced over time as described in the previous section, this characteristic of ISI distributions of antidromic firing cells persisted throughout the $4 \mathrm{~h}$ recording period (Fig. $5 C$ ).

\section{Reduced spike synchronization during subacute STN DBS}

A spike synchronization score was calculated to reflect the coincidence of neuronal firing across all simultaneously recorded M1 cells. Within each stimulation day in the awake resting state we observed a significant reduction in spike synchronization compared with the pre-DBS baseline in both animals (Fig. 6A). When all the stimulation days were combined, spike synchronization was also significantly decreased throughout the $4 \mathrm{~h}$ period of STN DBS in both animals (Fig. 6B; mixed-model ANOVA, Subject K, $F_{(6,9966)}=5.2, p=0.028$; Subject J, $\left.F_{(6,22780)}=8.1, p<0.0001\right)$.

To further demonstrate the cell-cell synchronization changes with STN DBS in each animal, pairwise spike synchronization analysis was performed and synchronization matrixes were generated. The spike synchronization matrixes from stimulation Day 1 illustrated changes in the pattern of pairwise synchronization in both subjects (Fig. 7A). The most salient change consistent in both animals was the reduction of spike synchronization of AntiNonAnti pairs, suggesting that the STN DBS may serve to "disconnect" antidromically activated cells from the rest of the M1 population. Combining the pairwise spike synchronization indexes in the averaged matrix from all the stimulation days, changes in spike synchronization in different cell-cell groups are shown in Figure $7 B$. The Anti-NonAnti spike synchronization decreased significantly during STN DBS in both animals (Wilcoxon test, Subject K, $\chi_{(3,4720)}^{2}=400.8, p<0.0001$; Subject J, $\left.\chi_{(3,2256)}^{2}=135.3, p<0.0001\right)$, whereas the NonAnti-NonAnti spike synchronization only decreased in Subject $\mathrm{K}\left(\chi_{(3,6108)}^{2}=\right.$ $194.7, p<0.0001)$. The increase in Anti-Anti spike synchronization at the first hour on DBS in Subject $\mathrm{K}$ was also significant $\left(\chi_{(3,844)}^{2}=37.3, p<0.0001\right)$. The pairwise spike synchronization in the $1 \mathrm{~h}$ post-DBS period returned to pre-DBS level for all of the cell-cell groups.

The proportion of cell pairs with increased or decreased cellcell spike synchronization in the fourth hour on DBS showed that more cell pairs had decreased spike synchronization (Fig. 7C). Moreover, decreased spike synchronization was observed with majority of Anti-NonAnti cell pairs (74.3\% in Subject K, 68.8\% in Subject J) and relatively more NonAnti-NonAnti cell pairs (61.0\% in Subject K, 54.6\% in Subject J), but slightly less AntiAnti cell pairs (48.8\% in Subject K, $42.1 \%$ in Subject J), in both subjects. These changes in the percentage of cell pairs with decreased spike synchronization are consistent with the changes in the cell-cell spike synchronization in all three groups (Fig. 7B).

Subacute GPi DBS was not collected in this study which precludes direct comparison of the effects of $4 \mathrm{~h}$ of STN and GPi DBS on spike synchronization. Analysis performed on neuronal data collected during acute 1 min STN and GPi DBS, however, revealed differential effects on spike synchronization. For acute STN DBS, a within-session comparison revealed significant reductions of spike synchronization in 75\% (3/4) and 100\% (5/5) of recording sessions in Subjects $\mathrm{K}$ and $\mathrm{J}$, respectively (pooled $t$ test, $p<0.05$ ). For acute GPi DBS, there were minimal changes detected in spike synchronization, with significant reductions observed in 20\% (2/5) of sessions in Subject K and none (0/5) in Subject J.

\section{Discussion}

Antidromic activation of $M 1$ as the primary mechanism underlying therapeutic DBS

A recent computational modeling study based on rodent anatomy reported that high-frequency STN stimulation caused robust, faithful propagation of action potentials throughout the axonal arbors of hyperdirect neurons (Anderson et al., 2018) 
A

\begin{tabular}{|c|c|c|c|c|}
\hline \multicolumn{5}{|c|}{ One-way Anova Test } \\
\hline Stimulation Day \# & F ratio & DF & Error DF & $\mathbf{p}$ \\
\hline $\mathbf{1}$ & 301.1 & 6 & 3096 & $<0.0001$ \\
\hline $\mathbf{2}$ & 197.0 & 5 & 2318 & $<0.0001$ \\
\hline $\mathbf{3}$ & 815.9 & 5 & 2418 & $<0.0001$ \\
\hline $\mathbf{4}$ & NA & NA & NA & NA \\
\hline $\mathbf{5}$ & 373.4 & 5 & 2110 & $<0.0001$ \\
\hline
\end{tabular}

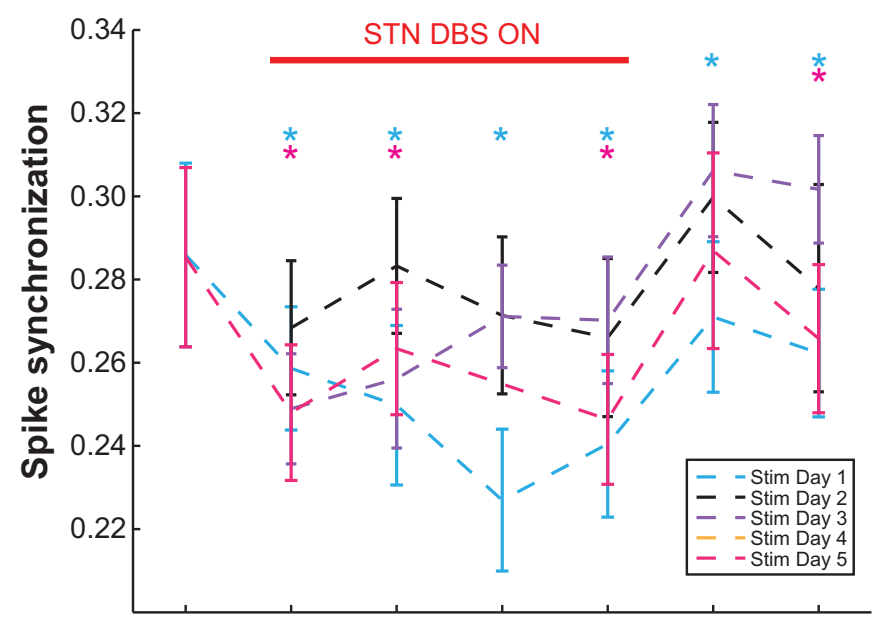

B

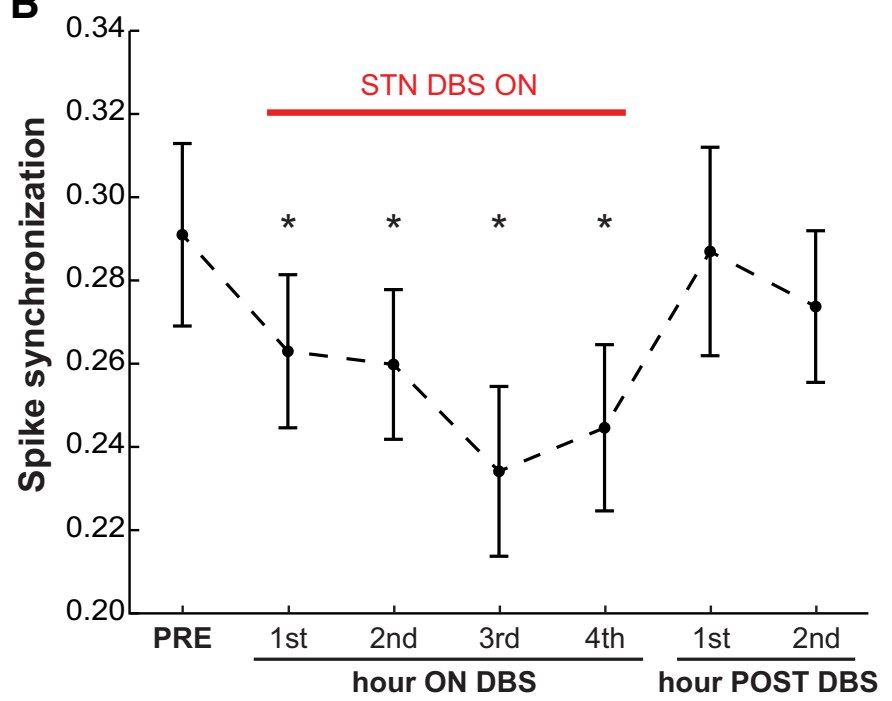

NHP J

\begin{tabular}{|c|c|c|c|c|}
\hline \multicolumn{5}{|c|}{ One-way Anova Test } \\
\hline Stimulation Day \# & F ratio & DF & Error DF & p \\
\hline $\mathbf{1}$ & 1037.5 & 5 & 4107 & $<0.0001$ \\
\hline $\mathbf{2}$ & 526.7 & 6 & 4847 & $<0.0001$ \\
\hline $\mathbf{3}$ & 409.7 & 6 & 4990 & $<0.0001$ \\
\hline $\mathbf{4}$ & 457.7 & 6 & 4441 & $<0.0001$ \\
\hline $\mathbf{5}$ & 291.9 & 5 & 4363 & $<0.0001$ \\
\hline
\end{tabular}
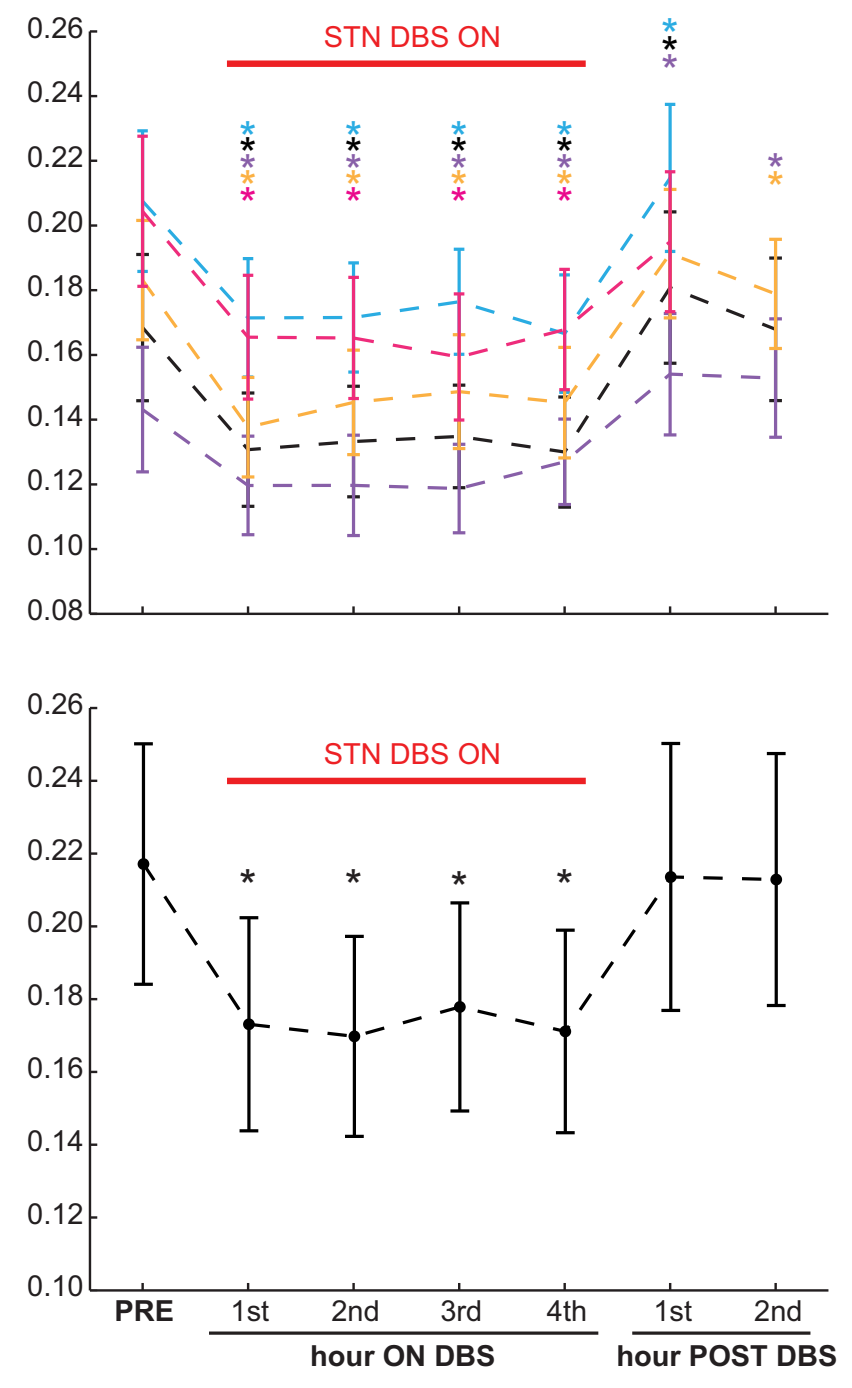

Time

Figure 6. Synchronization in populational unit activities decreased with therapeutic STN DBS. A, Spike synchronization scores for all simultaneously recorded M1 cells across multiple $4 \mathrm{~h}$ STN DBS stimulation days (color coded) in both animals are shown as mean \pm SD. Results of one-way ANOVA test for each session are shown in the tables (top). Following a significant one-way ANOVA test result, Dunnett's test with control $=$ PRE $\left({ }^{*} p<0.05\right)$ was performed for the sessions that had awake rest data in the PRE-DBS condition ( 2 for Subject K and 5 for Subject J), showing significant reduction of spike synchronization during STN DBS in each day. $\boldsymbol{B}$, Combined spike synchronization scores (mean \pm SD) from all the stimulation days that had the PRE-DBS condition are reduced during therapeutic STN DBS for both NHPs (mixed-model ANOVA, Subject K: $F_{(6,9966)}=5.2, p=0.028,2 \mathrm{~d}$; Subject J: $F_{(6,22780)}=8.1, p<0.0001,5$ d; followed by Dunnett's test with control $=$ $\operatorname{PRE}\left({ }^{*} p<0.05\right)$. The combined spike synchronization scores for the first and second hours post-DBS returned to the pre-DBS level (Dunnett's test with control $=$ PRE, $\left.p>0.05\right)$.

supporting previous experimental observations in rodents that M1 cells were antidromically activated during STN DBS (Li et al., 2012, 2014). The results of these and earlier studies have led to the hypothesis that antidromic activation of motor cortex cells via the hyperdirect pathway is a primary mechanism underlying the therapeutic effect of STN DBS (Dejean et al., 2009; Li et al., 2012,
2014). The same mechanism was also suspected to underlie the therapeutic effect of GPi DBS (Li et al., 2014). We sought to test this hypothesis in the MPTP nonhuman primate (NHP) model of PD where brain circuitry of NHPs is closer to that in humans and the size of the NHP brain allowed for implantation of DBS devices similar to those used in patients receiving DBS. This mon- 
A



1. Anti - Anti

2. Anti - NonAnti

NHP J
3. NonAnti - NonAnti

PRE DBS

Unit \#

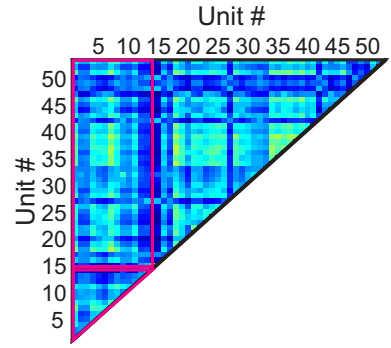

$\begin{array}{lllllll}5 & 10 & 15 & 20 & 25 & 30 & 35\end{array}$

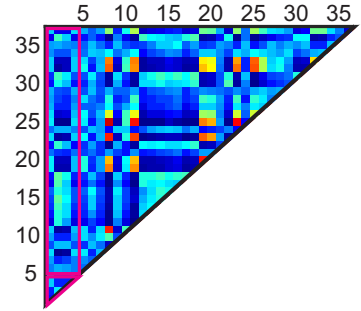

NHP K

B

C

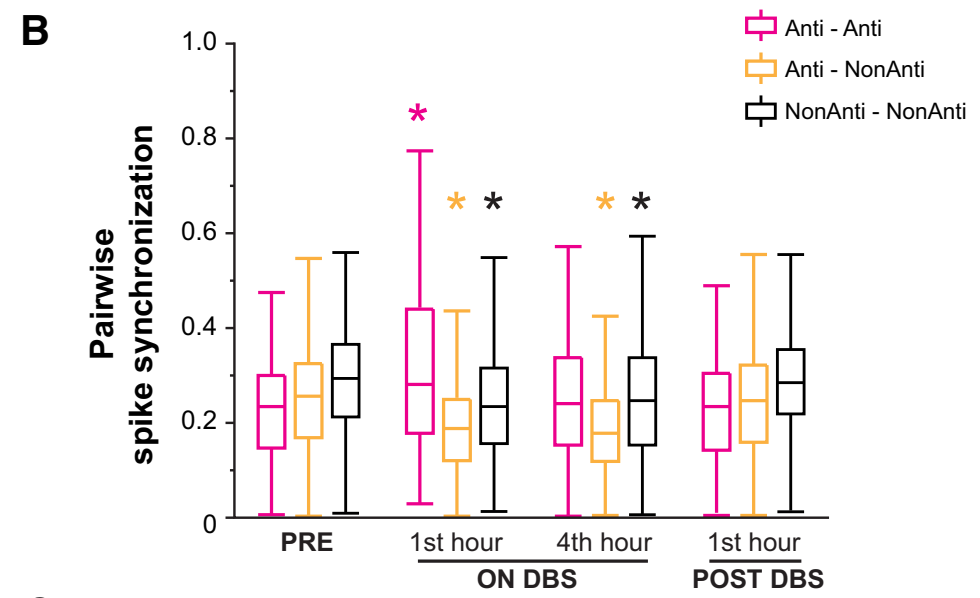

1st hour on DBS
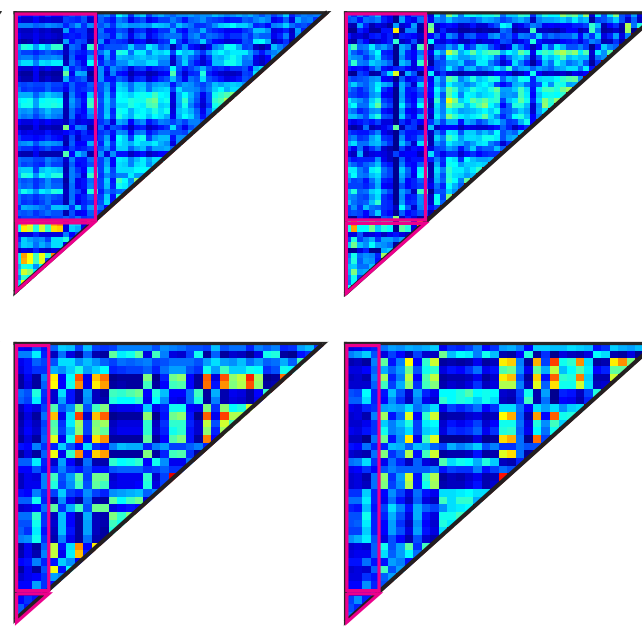

4th hour on DBS 1st hour post DBS
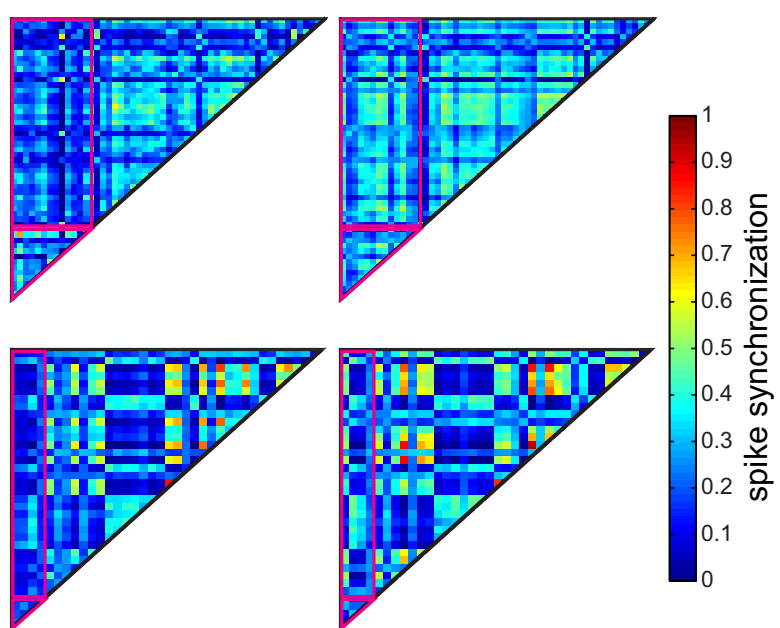

NHP J
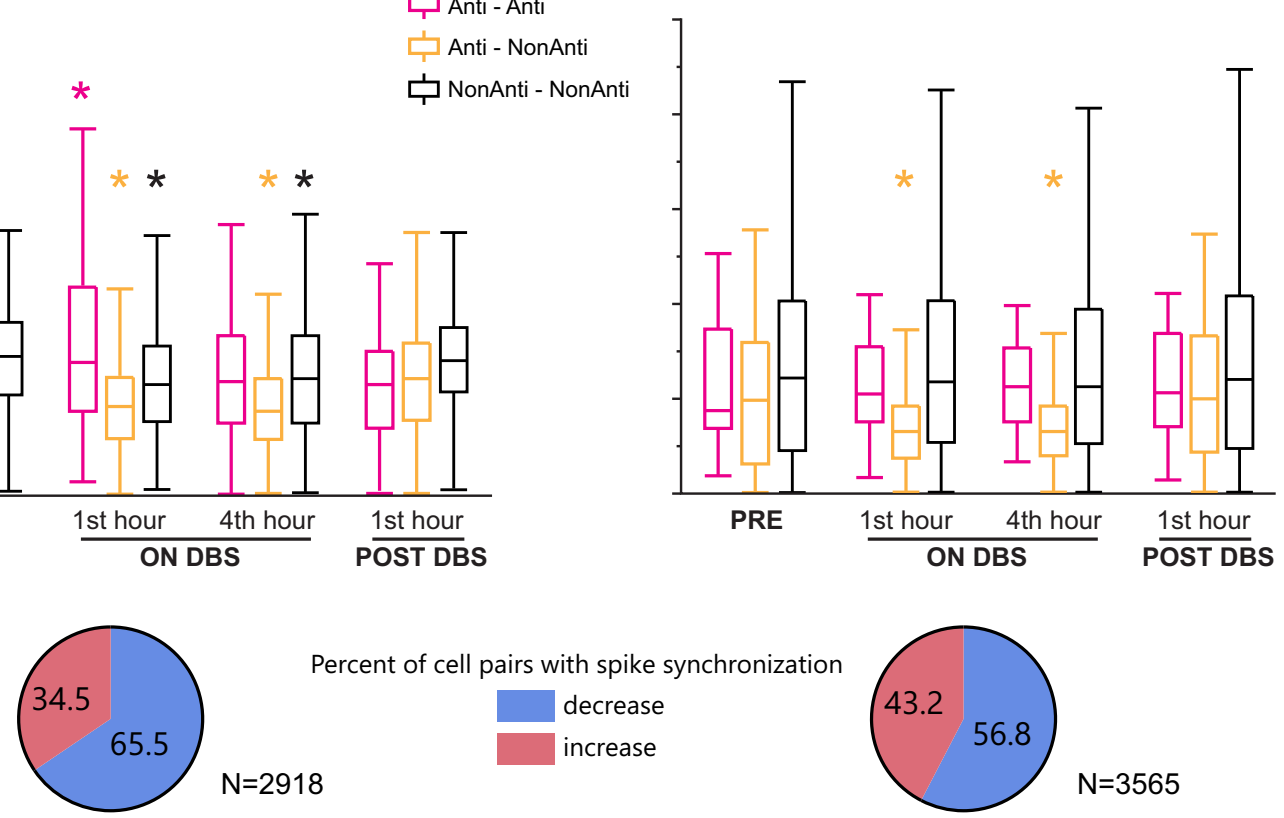

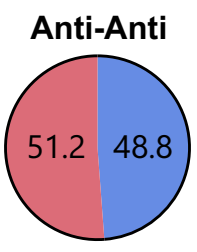

$\mathrm{N}=211$
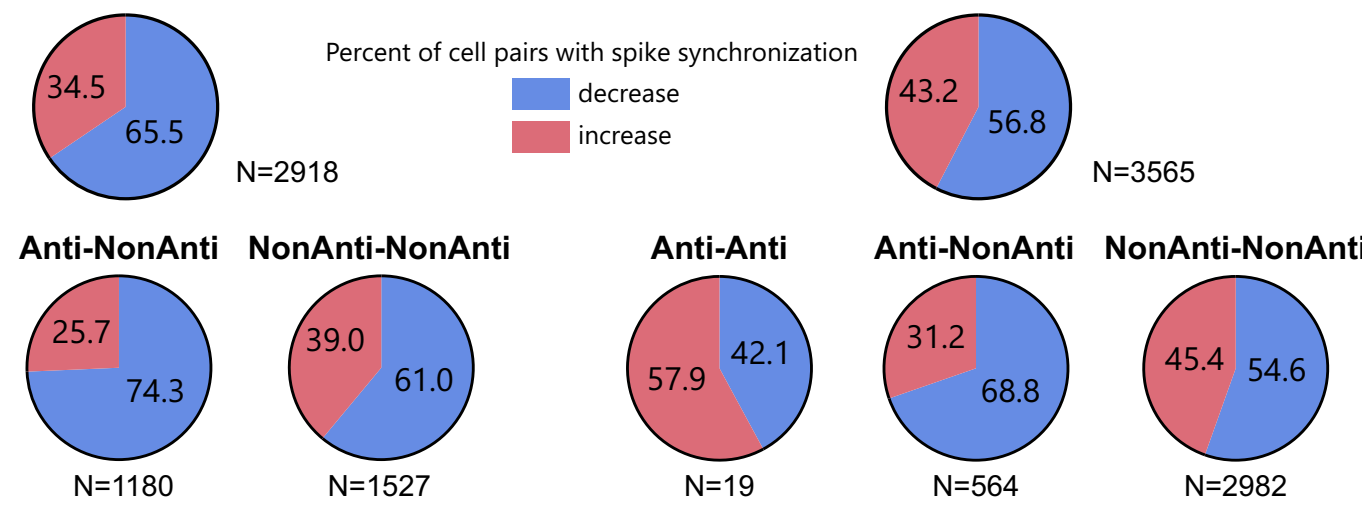

Figure 7. Pairwise spike synchronization of simultaneously recorded unit activities pre, during and post-therapeutic STN DBS in both NHPs. $A$, Averaged matrix illustration of the pattern of cell-cell synchronization changes with STN DBS from the first stimulation day in each animal. Matrices were generated based on the synchronization score calculated for each pair of cells. Fourteen (of 54) and four (of 30) antidromic classified neurons were identified in Subjects K and J, respectively, in these examples. Different pairs including antidromic to antidromic (Anti-Anti), antidromic to non-antidromic (Anti-NonAnti), non-antidromic to non-antidromic (NonAnti-NonAnti) neurons are labeled as shown in the left matrix pattern. $\boldsymbol{B}$, Pairwise spike synchronization from all the stimulation days that had awake rest data in the PRE-DBS condition (2 for Subject K and 5 for Subject J) for different groups (Anti-Anti, Anti-NonAnti and NonAnti-NonAnti) are shown. Anti-NonAnti pairwise spike synchronization was reduced by STN DBS in both subjects, whereas NonAnti-NonAnti spike synchronization was only reduced in Subject K. Anti-Anti spike synchronization did not change except for an increase at the first hour on STN DBS in Subject K. Wilcoxon test followed by Steel's test with control $=$ PRE. ${ }^{*} p<0.05$. C, Proportion of cell pairs with increased or decreased cell-cell spike synchronization scores, comparing pre-DBS period to fourth hour on-DBS, in all cell pairs (top) and in different groups (bottom). 
key model also produces motor signs similar to that in PD patients permitting behavioral assessments that also more closely reflect the human condition.

We observed antidromic activation of M1 cells during STN DBS in both monkeys suggesting activation of the hyperdirect pathway occurs with STN DBS in the NHP model of PD. A caveat to our observation however, is that despite similar clinical benefits with DBS in our two subjects, the proportion of recorded antidromic classified cells was dramatically different. Furthermore, although clinical improvement was maintained during subacute stimulation the strength of antidromic activation significantly decreased over time. It is possible that the difference in the percentage of M1 cells antidromically activated across animals were due to differences in DBS lead placement, such that M1 axons were preferentially activated in Subject K compared with J. The therapeutic levels for STN DBS were set $\sim 80 \%$ the level for internal capsule stimulation side effects; the fact that stimulation was lower in Subject K compared with Subject J (e.g., 0.3 vs 0.425 $\mathrm{mA}$ for acute DBS) is indeed suggestive of a lead location closer to the internal capsule in Subject K. If antidromic activation of M1 is important for STN DBS therapeutic effects, however, this study raises the question of how much if any antidromic activation is sufficient or necessary to impart that effect; the finding of similar therapeutic effects in both animals but $32 \%$ antidromic-classified cells in one animal and 6\% in the other suggests one of two things; either only a small proportion is required to elicit motor benefit or antidromic activation does not play a significant role in mediating the therapeutic effect of STN DBS.

Multiple studies in patients (Heywood and Gill, 1997; Levy et al., 2001; Rodriguez-Rojas et al., 2018) and animal models of PD (Bergman et al., 1990; Wichmann et al., 1994) have demonstrated that inactivation of the STN through pharmacological or ablative lesions can also alleviate parkinsonian motor signs. Although STN lesions would disrupt hyperdirect cortical-STN connectivity, they clearly would not produce activation of motor cortex via the hyperdirect pathway. Given that STN lesions and STN DBS produce similar therapeutic effects one might hypothesize they share at least some common mechanisms of action. However, although the former removes output from the lesioned structure the latter activates axons projecting from, projecting to, through and adjacent to the stimulated structure (Miocinovic et al., 2006; Johnson et al., 2008; Agnesi et al., 2013b). Nevertheless both could serve to ultimately disrupt the transmission of pathological information through the network just by different means (i.e., inactivating neuronal activity via lesion versus imposing more regular output on upstream/downstream nuclei via stimulation; Hashimoto et al., 2003; Xu et al., 2008; Agnesi et al., 2013a). It is possible that disrupting hyperdirect cortical-STN connectivity is important for therapeutic efficacy of STN interventions; however the primary mechanism of action of STN lesions and STN DBS common to both therapies (if one exists) is unlikely to be via direct activation of the hyperdirect pathway.

The necessity of hyperdirect activation for beneficial effects of GPi DBS was questioned by our finding of no antidromic classified cells in either animal during GPi DBS despite similar therapeutic benefits as STN DBS. This result is not surprising given that the GPi is not known to receive strong direct projections from M1, whereas the hyperdirect corticosubthalamic projects are well established by tracer studies (Nambu et al., 1996, 1997). A unique characteristic of our study was its within-subject design with animals implanted in both STN and GPi, which enabled clear demonstration of differences in M1 activation between targets. Our results support the conclusion by Miocinovic et al.
(2018), that antidromic activation of the hyperdirect pathway is unlikely to be the primary mechanism for achieving therapeutic DBS in the basal ganglia given the similar clinical effects achieved with STN and GP DBS (Miocinovic et al., 2018). It is important to note that while antidromic activation of M1 was not observed during GPi DBS this does not rule out a role for antidromic activation of M1 as a possible mechanism for STN DBS. We have previously reported different effects of STN to GPi DBS on thalamic activity in the cerebellar receiving areas of the motor thalamus (Xu et al., 2008; Muralidharan et al., 2017). The lack of such an effect during GPi DBS is consistent with the argument that although similar clinical benefit can occur with either target site the mechanisms by which they exert their therapeutic effect may differ.

\section{Antidromic activation of $M 1$ as a contributor to the therapeutic effect of STN DBS}

During subacute STN DBS, spike synchronization across all the simultaneously recorded neurons was reduced throughout the stimulation period (Fig. 6). The pairwise synchronization analysis (Fig. 7) suggests that the decreased spike synchronization between antidromic and non-antidromic neurons was the major contributor to the reduction in overall spike synchronization. This is supportive of the idea that antidromic activation serves to functionally disconnect those cells from the rest of the cortical cell population (Anderson et al., 2018), disrupting pathological network synchronization and leading to improved motor signs. Indeed, the decreased synchronization of antidromic classified neurons from the non-antidromic neurons was maintained throughout the stimulation period and paralleled the improvement in clinical rating scores. Interestingly, only a fraction of neurons fired antidromically (7-13\%) during subacute STN DBS in Subject J compared with Subject K (26-35\%), yet the change in population synchronization in both NHPs was similar and appeared to be driven by the change in synchronization between antidromic and non-antidromic cells. Moreover, the decrease in population spike synchronization persisted despite decreased robustness of antidromic firing over time (Fig. 4). These findings again bring up the question of how much antidromic activation is necessary to induce a cell population desynchronization and evoke therapeutic effects. We should also note that in this study we did not explore the relative change in synchronized oscillatory activity or connectivity changes within the basal ganglia thalamocortical circuit all of which are likely to play a role in mediating the therapeutic effect of DBS (Hashimoto et al., 2003; Meissner et al., 2005; de Hemptinne et al., 2015; Wang et al., 2018).

The time course of wash-in effects of DBS (on the scale of seconds to minutes) on individual motor signs has not been well characterized in PD patients, however in clinical practice it is observed that STN DBS can have a more rapid onset of effects compared with GPi DBS. An intriguing hypothesis to explain this difference is that direct modulation of motor cortical activity during STN DBS facilitates the rapid onset of symptom relief. It is notable that decreased spike synchronization was observed in most recording sessions during acute STN DBS but not GPi DBS. It important to note that the data presented here comparing STN to GPi acute DBS effects reflect motor cortical activity in the first minute after DBS is turned on, whereas our clinical ratings that show similar therapeutic effects were collected several minutes later. Additional studies are necessary to determine whether subacute GPi DBS produces the same decrease in synchronization that is observed with subacute STN DBS. To better address these questions regarding the relative time course of behavioral and 
neuronal effects of STN versus GPi DBS, future studies will require continuous neural recordings combined with methods to monitor and quantify individual motor signs on a moment by moment basis.

\section{Limitations}

One potential limitation of this study is that although both animals were implanted in the arm area of M1 with identical Utah arrays we cannot rule out differences in M1 cell sampling, such that similar antidromic firing was actually occurring in both subjects but was by chance under-sampled in Subject J. Further investigation in additional animals combining subject-specific fiber tractography and volume of tissue activation models with electrophysiology measures across a range of stimulation settings that do and do not produce therapeutic effects will be needed to further elucidate the relationship, if one exists, between activation of M1 axonal projections and therapeutic effects of STN DBS. Subacute (4 h) GPi DBS was not evaluated in these animals; to further study the similarities and differences in STN and GPi DBS mechanisms of action (e.g., decreases in spike synchronization), future studies should incorporate within-subject comparisons of neuronal population spiking activity during long-term STN and GPi DBS.

\section{Concluding remarks}

Although this study does not rule out a contribution of antidromic activation in M1 during STN DBS to the acute improvement in motor signs, the difference in observed antidromic activity between animals, and lack of antidromic activation during GPi DBS, raise questions about its hypothesized role as the primary mechanism for achieving therapeutic DBS in the basal ganglia. Given the network wide dysfunction of cortical and subcortical neuronal activity in PD it is difficult to state that changes in any one nodal point (e.g., M1) of the basal gangliathalamocortical (BGTC) circuit is responsible for the motor dysfunction observed in PD (Lalo et al., 2008; Jahanshahi et al., 2010; Canessa et al., 2016). It can be argued that it is the sum of these changes leading to alterations network connectivity (Kahan et al., 2014) rather than the activity of a specific node. Although DBS in the STN and GPi may have differential effects on M1 spiking activity as we have shown in this study, both may ultimately serve to disrupt pathological network activity or move the altered patterns toward that observed in the normal state. Future studies investigating the impact of therapeutic DBS on neuronal populations across multiple sites within the BGTC circuit recorded simultaneously both at rest and during movement will be critical in addressing these questions.

\section{References}

Agnesi F, Connolly AT, Baker KB, Vitek JL, Johnson MD (2013a) Deep brain stimulation imposes complex informational lesions. PLoS One 8:e74462.

Agnesi F, Johnson MD, Vitek JL (2013b) Deep brain stimulation: how does it work? Handb Clin Neurol 116:39-54.

Anderson RW, Farokhniaee A, Gunalan K, Howell B, McIntyre CC (2018) Action potential initiation, propagation, and cortical invasion in the hyperdirect pathway during subthalamic deep brain stimulation. Brain Stimul 11:1140-1150.

Benabid AL, Pollak P, Louveau A, Henry S, de Rougemont J (1987) Combined (thalamotomy and stimulation) stereotactic surgery of the VIM thalamic nucleus for bilateral parkinson disease. Appl Neurophysiol 50: 344-346.

Bergman H, Wichmann T, DeLong MR (1990) Reversal of experimental parkinsonism by lesions of the subthalamic nucleus. Science 249:14361438.

Burchiel KJ, Anderson VC, Favre J, Hammerstad JP (1999) Comparison of pallidal and subthalamic nucleus deep brain stimulation for advanced Parkinson's disease: results of a randomized, blinded pilot study. Neurosurgery 45:1375-1384.

Canessa A, Pozzi NG, Arnulfo G, Brumberg J, Reich MM, Pezzoli G, Ghilardi MF, Matthies C, Steigerwald F, Volkmann J, Isaias IU (2016) Striatal dopaminergic innervation regulates subthalamic beta-oscillations and cortical-subcortical coupling during movements: preliminary evidence in subjects with Parkinson's disease. Front Hum Neurosci 10:611.

de Hemptinne C, Swann NC, Ostrem JL, Ryapolova-Webb ES, San Luciano M, Galifianakis NB, Starr PA (2015) Therapeutic deep brain stimulation reduces cortical phase-amplitude coupling in Parkinson's disease. Nat Neurosci 18:779-786.

Dejean C, Hyland B, Arbuthnott G (2009) Cortical effects of subthalamic stimulation correlate with behavioral recovery from dopamine antagonist induced akinesia. Cereb Cortex 19:1055-1063.

Dostrovsky JO, Levy R, Wu JP, Hutchison WD, Tasker RR, Lozano AM (2000) Microstimulation-induced inhibition of neuronal firing in human globus pallidus. J Neurophysiol 84:570-574.

Escobar Sanabria D, Johnson LA, Nebeck SD, Zhang J, Johnson MD, Baker KB, Molnar GF, Vitek JL (2017) Parkinsonism and vigilance: alteration in neural oscillatory activity and phase-amplitude coupling in the basal ganglia and motor cortex. J Neurophysiol 118:2654-2669.

Follett KA, Weaver FM, Stern M, Hur K, Harris CL, Luo P, Marks WJ Jr, Rothlind J, Sagher O, Moy C, Pahwa R, Burchiel K, Hogarth P, Lai EC, Duda JE, Holloway K, Samii A, Horn S, Bronstein JM, Stoner G, et al. (2010) Pallidal versus subthalamic deep-brain stimulation for Parkinson's disease. N Engl J Med 362:2077-2091.

Gradinaru V, Mogri M, Thompson KR, Henderson JM, Deisseroth K (2009) Optical deconstruction of parkinsonian neural circuitry. Science 324: 354-359.

Hashimoto T, Elder CM, Okun MS, Patrick SK, Vitek JL (2003) Stimulation of the subthalamic nucleus changes the firing pattern of pallidal neurons. J Neurosci 23:1916-1923.

Heywood P, Gill SS (1997) Bilateral dorsolateral subthalamotomy for advanced Parkinson's disease. Lancet 350:1224.

Jahanshahi M, Jones CR, Zijlmans J, Katzenschlager R, Lee L, Quinn N, Frith CD, Lees AJ (2010) Dopaminergic modulation of striato-frontal connectivity during motor timing in Parkinson's disease. Brain 133:727-745.

Johnson MD, Miocinovic S, McIntyre CC, Vitek JL (2008) Mechanisms and targets of deep brain stimulation in movement disorders. Neurotherapeutics 5:294-308.

Kahan J, Urner M, Moran R, Flandin G, Marreiros A, Mancini L, White M, Thornton J, Yousry T, Zrinzo L, Hariz M, Limousin P, Friston K, Foltynie $\mathrm{T}$ (2014) Resting state functional MRI in Parkinson's disease: the impact of deep brain stimulation on "effective" connectivity. Brain 137:11301144.

Kreuz T, Mulansky M, Bozanic N (2015) SPIKY: a graphical user interface for monitoring spike train synchrony. J Neurophysiol 113:3432-3445.

Kuriakose R, Saha U, Castillo G, Udupa K, Ni Z, Gunraj C, Mazzella F, Hamani C, Lang AE, Moro E, Lozano AM, Hodaie M, Chen R (2010) The nature and time course of cortical activation following subthalamic stimulation in Parkinson's disease. Cereb Cortex 20:1926-1936.

Lalo E, Thobois S, Sharott A, Polo G, Mertens P, Pogosyan A, Brown P (2008) Patterns of bidirectional communication between cortex and basal ganglia during movement in patients with Parkinson disease. J Neurosci 28: 3008-3016.

Levy R, Lang AE, Dostrovsky JO, Pahapill P, Romas J, Saint-Cyr J, Hutchison WD, Lozano AM (2001) Lidocaine and muscimol microinjections in subthalamic nucleus reverse parkinsonian symptoms. Brain 124:21052118.

Li Q, Ke Y, Chan DC, Qian ZM, Yung KK, Ko H, Arbuthnott GW, Yung WH (2012) Therapeutic deep brain stimulation in parkinsonian rats directly influences motor cortex. Neuron 76:1030-1041.

Li Q, Qian ZM, Arbuthnott GW, Ke Y, Yung WH (2014) Cortical effects of deep brain stimulation: implications for pathogenesis and treatment of parkinson disease. JAMA Neurol 71:100-103.

Maynard EM, Nordhausen CT, Normann RA (1997) The Utah intracortical electrode array: a recording structure for potential brain-computer interfaces. Electroencephalogr Clin Neurophysiol 102:228-239.

Meissner W, Leblois A, Hansel D, Bioulac B, Gross CE, Benazzouz A, Boraud $\mathrm{T}$ (2005) Subthalamic high frequency stimulation resets subthalamic firing and reduces abnormal oscillations. Brain 128:2372-2382. 
Miocinovic S, Parent M, Butson CR, Hahn PJ, Russo GS, Vitek JL, McIntyre CC (2006) Computational analysis of subthalamic nucleus and lenticular fasciculus activation during therapeutic deep brain stimulation. J Neurophysiol 96:1569-1580.

Miocinovic S, Noecker AM, Maks CB, Butson CR, McIntyre CC (2007) Cicerone: stereotactic neurophysiological recording and deep brain stimulation electrode placement software system. Acta Neurochir Suppl 97:561-567.

Miocinovic S, de Hemptinne C, Chen W, Isbaine F, Willie JT, Ostrem JL, Starr PA (2018) Cortical potentials evoked by subthalamic stimulation demonstrate a short latency hyperdirect pathway in humans. J Neurosci 38:9129-9141.

Monakow KH, Akert K, Künzle H (1978) Projections of the precentral motor cortex and other cortical areas of the frontal lobe to the subthalamic nucleus in the monkey. Exp Brain Res 33:395-403.

Mulansky M, Bozanic N, Sburlea A, Kreuz T (2015) A guide to timeresolved and parameter-free measures of spike train synchrony. 2015 International Conference on Event-based Control, Communication, and Signal Processing (EBCCSP). Krakow, Poland, June.

Muralidharan A, Zhang J, Ghosh D, Johnson MD, Baker KB, Vitek JL (2017) Modulation of neuronal activity in the motor thalamus during GPi-DBS in the MPTP nonhuman primate model of Parkinson's disease. Brain Stimul 10:126-138.

Nambu A, Takada M, Inase M, Tokuno H (1996) Dual somatotopical representations in the primate subthalamic nucleus: evidence for ordered but reversed body-map transformations from the primary motor cortex and the supplementary motor area. J Neurosci 16:2671-2683.

Nambu A, Tokuno H, Inase M, Takada M (1997) Corticosubthalamic input zones from forelimb representations of the dorsal and ventral divisions of the premotor cortex in the macaque monkey: comparison with the input zones from the primary motor cortex and the supplementary motor area. Neurosci Lett 239:13-16.

Rodriguez-Rojas R, Carballo-Barreda M, Alvarez L, Guridi J, Pavon N,
Garcia-Maeso I, Mací As R, Rodriguez-Oroz MC, Obeso JA (2018) Subthalamotomy for Parkinson's disease: clinical outcome and topography of lesions. J Neurol Neurosurg Psychiatry 89:572-578.

Rousche PJ, Normann RA (1992) A method for pneumatically inserting an array of penetrating electrodes into cortical tissue. Ann Biomed Eng 20:413-422.

Sanders TH, Jaeger D (2016) Optogenetic stimulation of cortico-subthalamic projections is sufficient to ameliorate bradykinesia in 6-ohda lesioned mice. Neurobiol Dis 95:225-237.

Vitek JL, Zhang J, Hashimoto T, Russo GS, Baker KB (2012) External pallidal stimulation improves parkinsonian motor signs and modulates neuronal activity throughout the basal ganglia thalamic network. Exp Neurol 233:581-586.

Walker HC, Huang H, Gonzalez CL, Bryant JE, Killen J, Cutter GR, Knowlton RC, Montgomery EB, Guthrie BL, Watts RL (2012) Short latency activation of cortex during clinically effective subthalamic DBS for Parkinson disease. Mov Disord 27:864-873.

Wang DD, de Hemptinne C, Miocinovic S, Ostrem JL, Galifianakis NB, San Luciano M, Starr PA (2018) Pallidal deep-brain stimulation disrupts pallidal beta oscillations and coherence with primary motor cortex in Parkinson's disease. J Neurosci 38:4556-4568.

Wichmann T, Bergman H, DeLong MR (1994) The primate subthalamic nucleus: III. Changes in motor behavior and neuronal activity in the internal pallidum induced by subthalamic inactivation in the MPTP model of Parkinsonism. J Neurophysiol 72:521-530.

Xu W, Russo GS, Hashimoto T, Zhang J, Vitek JL (2008) Subthalamic nucleus stimulation modulates thalamic neuronal activity. J Neurosci 28:11916-11924.

Zhang J, Wang ZI, Baker KB, Vitek JL (2012) Effect of globus pallidus internus stimulation on neuronal activity in the pedunculopontine tegmental nucleus in the primate model of Parkinson's disease. Exp Neurol 233:575580. 